Provided for non-commercial research and education use. Not for reproduction, distribution or commercial use.

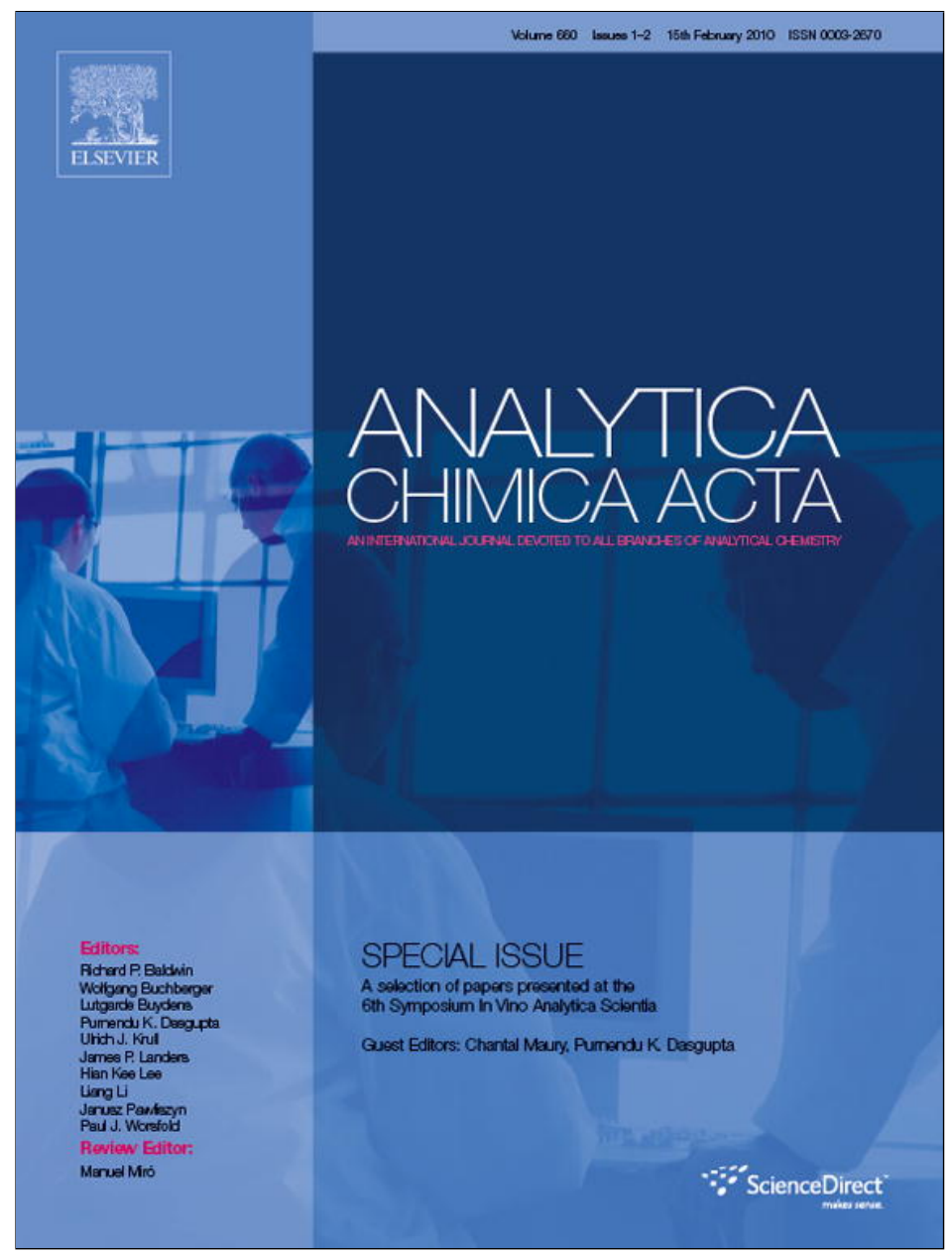

This article appeared in a journal published by Elsevier. The attached copy is furnished to the author for internal non-commercial research and education use, including for instruction at the authors institution and sharing with colleagues.

Other uses, including reproduction and distribution, or selling or licensing copies, or posting to personal, institutional or third party websites are prohibited.

In most cases authors are permitted to post their version of the article (e.g. in Word or Tex form) to their personal website or institutional repository. Authors requiring further information regarding Elsevier's archiving and manuscript policies are encouraged to visit:

http://www.elsevier.com/copyright 


\title{
Development of a fractionation method for the detection and identification of oak ellagitannins in red wines
}

\author{
Ignacio García-Estévez, M. Teresa Escribano-Bailón*, Julián C. Rivas-Gonzalo, Cristina Alcalde-Eon \\ Grupo de Investigación en Polifenoles (GIP), Unidad de Nutrición y Bromatología, Facultad de Farmacia, Universidad de Salamanca, \\ Campus Miguel de Unamuno, 37007 Salamanca, Spain
}

\section{A R T I C L E I N F O}

\section{Article history:}

Received 29 July 2009

Received in revised form 5 October 2009

Accepted 10 October 2009

Available online 17 October 2009

\section{Keywords:}

Oak ellagitannins

Red wine

High performance liquid

chromatography-diode array

detection-mass spectrometry

Fractionation

Characterisation

\begin{abstract}
A B S T R A C T
During maturation and ageing in oak barrels wines improve their organoleptic properties. Ellagitannins can be released from wood to the wine and be involved in oxidation reactions and seem to influence the astringency and colour properties of the wine.

Nevertheless, the ellagitannins levels are lower than those of other wine constituents and, consequently, they are not easily detected. This study has developed a two-step fractionation method consisting of a solid phase extraction in C-18 Sep-Pak ${ }^{\circledR}$ cartridges followed by size exclusion chromatography in hand-packed Sephadex LH-20 minicolumn for the detection of oak ellagitannins in different types of wines. An HPLC method has also been developed which allows the separation of compounds with the same $m / z$ ratios, facilitating the ellagitannin identification by means of the mass spectrometric analyses. The main oak ellagitannins (grandinin, vescalagin, roburin E and castalagin) were isolated, detected separately and identified in a spiked wine and in three real ones, proving the usefulness of the fractionation method.
\end{abstract}

(ㄷ) 2009 Elsevier B.V. All rights reserved.

\section{Introduction}

During maturation and ageing of wines in oak barrels different substances can be released from the wood to the wine and can take part in several reactions which might produce both qualitative and quantitative changes in the wine affecting its organoleptic properties such as colour and astringency [1-3]. The ellagitannins (hydrolysable tannins) are among these compounds. In oak heartwood they may represent $10 \%$ of the dry weight and are responsible for the high durability of this wood [4].

The most representative structures of ellagic tannins are vescalagin and castalagin, two isomers first isolated and described by Mayer [5]. Lyxose/xylose derivatives (grandinin and roburin E, respectively) and dimeric forms (roburins A, B, C, D) have also been described [6,7]. These compounds are extracted from wood to wine owing to the hydro-alcoholic nature of the wine. The extraction depends mainly on the pool of potential extractable compounds that are originally present in the barrel oak wood and on the conditions and duration of the winemaking process [8].

The content of the ellagitannins in oak wood is influenced by several factors [8]. On the one hand, the oak species (e.g., Quercus robur L., Quercus petraea (Matt.) Liebl., Q. alba L.), the geographical origin (e.g., American or European oak) $[9,10]$, the age [11] or

\footnotetext{
* Corresponding author. Tel.: +34 923294537; fax: +34 923294515.

E-mail address: escriban@usal.es (M.T. Escribano-Bailón).
}

the sylvocultural treatment of the tree, on the other, the processing of wood in cooperage that changes its chemical composition. The method to obtain the staves, the method of seasoning (natural or artificial, length, location, etc.) or the degree of toasting (no-toasting, light, medium or heavy toasting) have an important influence on the ellagitannins' content in oak [12,13]. As a result of all these treatments the ellagitannins can be oxidized and can form polymers and copolymers with cell-wall components. Thus, their solubility would be reduced [14]. Moreover, the age of the barrels is also important, since it has been reported that the levels of extracted ellagitannins are much lower in old barrels [15].

The ellagitannins possess several hydroxyl functions in ortho positions at the periphery of their molecular structures (Fig. 1). For this reason, in wine, they are involved in oxidation reactions, acting as consumers of oxygen which induce an increased oxidizing power that finally causes a large quantity of peroxides and acetaldehyde to be produced by oxidation [1]. Thus, ellagic tannins may take part in the changes of colour during maturation and ageing of wine, helping to improve colour stability and also protecting it against oxidation $[1,16,17]$. Oak wood ellagitannins can also affect the astringency of the wine, since, as procyanidins, they have the ability to precipitate proteins, in particular the salivary proteins in the oral cavity $[1,2,18]$.

During maturation and ageing of wine in oak barrels, ellagitannins are solubilised by wine and diffuse through the wood. Once in the wine the ester bonds of the ellagitannins may be easily 


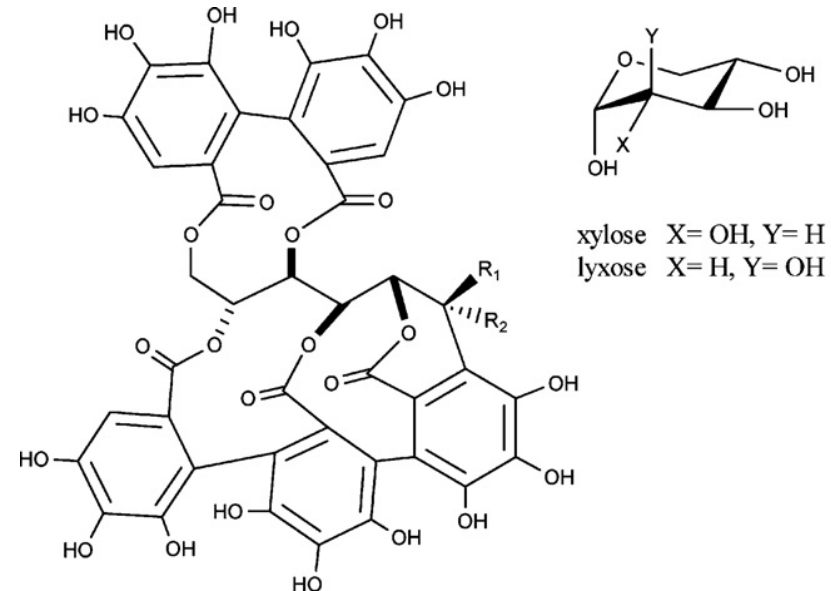

(1) Grandinin $R_{1}=$ lyxose, $R_{2}=H$

(3) Roburin E $R_{1}=$ xylose, $R_{2}=H$

(2) Vescalagin $\mathrm{R}_{1}=\mathrm{OH}, \mathrm{R}_{2}=\mathrm{H}$

(4) Castalagin $\mathrm{R}_{1}=\mathrm{H}, \mathrm{R}_{2}=\mathrm{OH}$

Fig. 1. Structure of the main oak ellagitannins.

hydrolysed giving rise, by rearrangement of the HHDP (hexahydroxydiphenic) residue, to ellagic acid. The ellagitannins might also be oxidised in its HHDP residue. However, in this case, the product of its degradation would not be ellagic acid $[15,19]$. Furthermore, due to their high reactivity, ellagitannins are involved in many other processes during the ageing of wine. Consequently, their levels in wine are much lower than those of other wine constituents and, as a result, they are not easily detected in the chromatograms even recorded at their maximum wavelength. Moreover, their structural similarities make their chromatographic separation difficult in reversed phases as well as their identification by means of the spectral features (identical UV-vis spectra, pseudo-molecular ions with the same $m / z$ ratios).

There are few studies whose objective has been the qualitative and quantitative determination of the ellagitannin composition in wines. Some of them only determine the total concentration of these compounds [20] following a procedure usually employed in the quantification of total ellagitannins in wood [14,21], which is based on acid hydrolysis of the ellagitannins and subsequent quantification of the free ellagic acid released. Nevertheless, when the studies had the objective of the qualitative and quantitative characterisation of the wine samples [22] and, in some cases grapes [23], a fractionation step was always required. In the existing methods the high volume of sample required, the complexity of some of the stages and the presence of targeted compounds in more than a single fraction can be considered as the main drawbacks.

The main objective of this study was to obtain a fractionation method which would allow the isolation of the original oak ellagitannins from other wine constituents in order to make their detection and quantification in wine samples possible. This study also had the objective of obtaining an HPLC method which would separate compounds with the same $m / z$ ratios in order to facilitate their identification and quantification by means of mass spectrometric analyses.

\section{Experimental}

\subsection{Isolation of the ellagitannins from oak chips}

One hundred grams of medium toasted chips obtained from Q. petraea (Matt.) Liebl. wood were macerated overnight in $2.5 \mathrm{~L}$ of a hydromethanolic solution (1:1) with constant stirring. Chips were extracted twice with the same protocol and both extracts were gathered and concentrated under reduced pressure in order to remove the methanol. Then the aqueous extract was first cleaned three times with diethyl ether and three times with ethyl acetate in order to remove other extracted oak compounds which might have interfered in further isolation steps. Once cleaned, the extract was concentrated under reduced pressure in a rotary evaporator to a final volume of $800 \mathrm{~mL}$ and half of it was loaded in a Sephadex LH20 (Sigma-Aldrich, St. Louis, MO, USA) column $(55 \mathrm{~mm} \times 270 \mathrm{~mm})$ previously conditioned with a methanol:water (40:60) solution. The elution was carried out with methanol:acidified water $(\mathrm{pH}$ 4.5 , acetic acid) (40:60). Several fractions were obtained and their composition was determined by means of HPLC-DAD-MS. Among them those containing the major oak ellagitannins (grandinin (1), vescalagin (2), roburin E (3), castalagin (4), Fig. 1) were selected and each ellagitannin isolated from them by means of semi-preparative HPLC. The equipment used consisted of a Waters 600E pump and Waters 600E pump controller with a Supelco C-18 reversedphase, $5 \mu \mathrm{m}(250 \mathrm{~mm} \times 10 \mathrm{~mm})$ column. The solvent employed in the elution was a solution of $2.5 \%$ acetic acid in ultrapure water which was pumped at a constant flow of $1.5 \mathrm{~mL} \mathrm{~min}^{-1}$. Detection was carried out at $240 \mathrm{~nm}$ with a Waters 486 tunable absorbance detector. The different fractions were then analysed by HPLC-DAD-MS (instruments and methods described in Section 2.3) and those with purities higher than $95 \%$ of one ellagitannin were gathered, concentrated under reduced pressure and freeze-dried. The identities of the ellagitannins were assigned from the data obtained in the HPLC-DAD-MS and in the ${ }^{1} \mathrm{H}$ and ${ }^{13} \mathrm{C}$ NMR analyses $[2,21,24,25]$. The final purities of the powders were determined from the results of the HPLC-DAD-MS analyses. All the solvents employed were analytical grade and were purchased from Prolabo ${ }^{\circledR}$ (BDH) VWR International (Briare, France). The ultrapure water was obtained from a Direct- $\mathrm{Q}^{\mathrm{TM}}$ water purification system equipped with a Millipak ${ }^{\circledR} 40(22 \mu \mathrm{m})$ filter unit (Millipore, Billerica, MA, USA).

\subsection{Development of the fractionation method}

Three millilitres of a red wine elaborated from Vitis vinifera $\mathrm{L}$. cv. "Tempranillo" grapes was spiked with solutions of the pure oak ellagitannins (grandinin, vescalagin, roburin E, castalagin). The concentration of each of these ellagitannins in the spiked wine was about $25 \mathrm{mg} \mathrm{L}^{-1}$.

The spiked wine was then concentrated under reduced pressure in order to remove ethanol and diluted with a solution of $2.5 \%$ acetic acid in water up to a final volume of $5 \mathrm{~mL}$. Then, it was fractionated in a two-step procedure similar to that described by Lee et al. [23] for the isolation of ellagic acid conjugates and other polyphenolics in muscadine grapes. Nevertheless, and due to the differences in the targeted compounds in each study, some modifications were made (Fig. 2).

The sample was first applied onto a Waters C-18 Sep-Pak ${ }^{\circledR}$ (500 mg) cartridge (Millipore Corp., Milford, MA, USA), previously activated with methanol and equilibrated with $2.5 \%$ acetic acid in water. The first fraction (fraction $a$ ) was collected from the moment of the application of the sample onto the C-18 cartridge to the end of the loading step and during the elution with $5 \mathrm{~mL}$ of $2.5 \%$ acetic acid in water. The second fraction (fraction $b$ ) was eluted with $5 \mathrm{~mL}$ of ethyl acetate and the third (fraction $c$ ), with $5 \mathrm{~mL}$ of methanol. Fractions $a$ and $b$ were evaporated under reduced pressure and re-dissolved in $2.5 \%$ acetic acid to a final volume of $2 \mathrm{~mL}$. Fraction $c$ was also evaporated in order to remove methanol and was re-dissolved in $2.5 \%$ acetic acid to a final volume of $5 \mathrm{~mL}$. Fraction $a$ was subsequently submitted to another fractionation in a hand-packed Sephadex LH-20 minicolumn $(10 \mathrm{~mm} \times 30 \mathrm{~mm})$ previously activated with methanol and equilibrated with ultrapure water. In this second fractionation, three different eluents were employed obtaining four eluates as follows: the first eluate (fraction 
1) SPE in a C-18 Sep-pak ${ }^{\circledR}$ cartridge

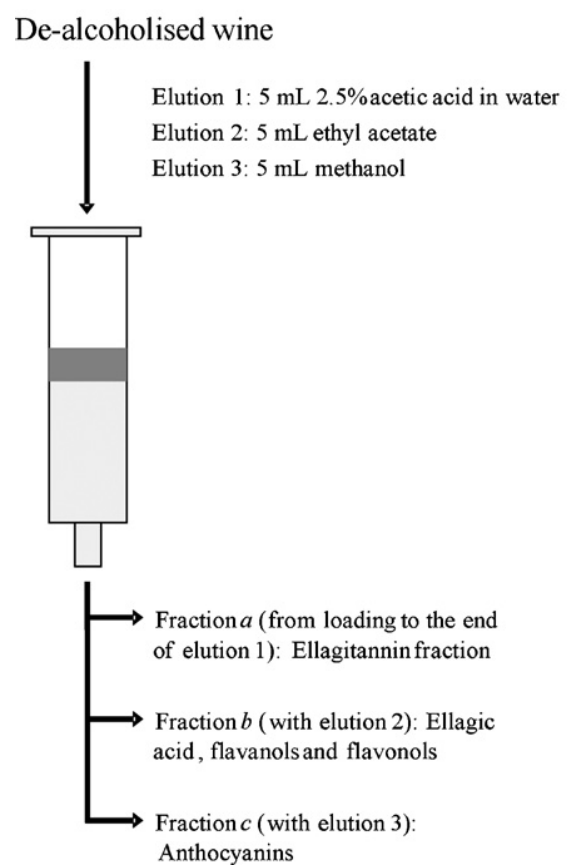

2) Size exclusion chromatography of fraction $a$ in Sephadex LH-20

Fraction $a(2 \mathrm{~mL})$

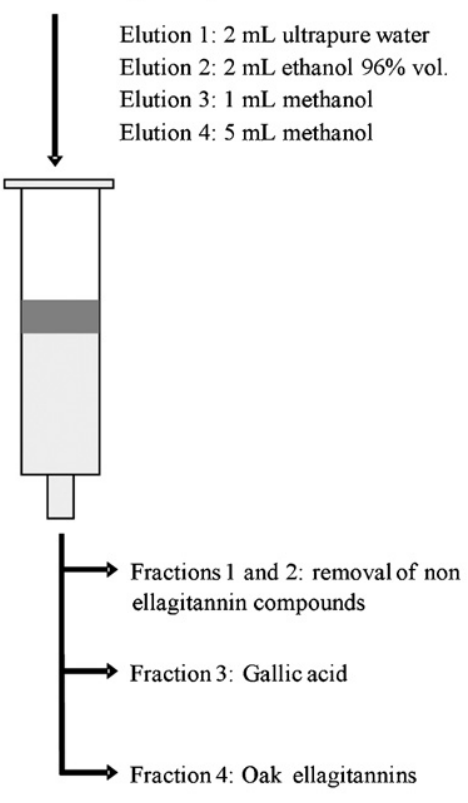

Fig. 2. Scheme of the two-step fractionation method developed in the present study for the separation of the oak ellagitannins' fraction from red wines.

1) was obtained with $2 \mathrm{~mL}$ of ultrapure water, the second (fraction 2), with $2 \mathrm{~mL}$ of $100 \%$ ethanol (96\% vol.), the third (fraction 3 ) with $1 \mathrm{~mL}$ of $100 \%$ methanol and the last (fraction 4), with $5 \mathrm{~mL}$ of $100 \%$ methanol. All these eluates were evaporated under reduced pressure and re-dissolved in $2.5 \%$ acetic acid to a final volume of $2 \mathrm{~mL}$. All the fractions obtained in both fractionations were analysed by HPLC-DAD-MS in order to localise the oak ellagitannins.

\subsection{HPLC-DAD-MS analyses}

HPLC-DAD analyses were performed in a Hewlett-Packard 1100 series liquid chromatograph. An AQUA C-18 reversed-phase, $5 \mu \mathrm{m}$, $150 \mathrm{~mm} \times 4.6 \mathrm{~mm}$ column (Phenomenex ${ }^{\circledR}$, Torrance, CA, USA) thermostatted at $35^{\circ} \mathrm{C}$ was used. Two HPLC methods were developed for the analyses of different fractions.

Method 1 (for the ellagitannins analyses): The ellagitannin-rich fraction (fraction $a$ ) and the fractions obtained in the size exclusion chromatography with Sephadex LH-20 (fractions 1-4) were analysed with this method. The HPLC-DAD conditions were optimised with oak extracts rich in the four main ellagitannins. The solvents used were: (A) an aqueous solution (2.5\%) of acetic acid, (B) $100 \%$ HPLC-grade isopropanol (HiPerSolv ${ }^{\circledR}$ Chromanorm, BDH Prolabo, VWR International, Briare, France) and (C) 100\% HPLC-grade methanol (HiPerSolv ${ }^{\circledR}$ Chromanorm, BDH Prolabo, VWR International, Briare, France). In order to solve the problem of the global shortage of acetonitrile, different mixtures of isopropanol and methanol were tested. The following gradient was finally established: isocratic $100 \% \mathrm{~A}$ for $14 \mathrm{~min}$ at flow $0.4 \mathrm{~mL} \mathrm{~min}^{-1}$, from 0.4 to $0.5 \mathrm{~mL} \mathrm{~min}^{-1}$ for $1 \mathrm{~min}$, from $100 \% \mathrm{~A}$ to $90 \% \mathrm{~A}, 2 \% \mathrm{~B}, 8 \% \mathrm{C}$ for 5 min, from $90 \%$ A, $2 \%$ B, $8 \%$ C to $60 \%$ A, $8 \%$ B, 32\% C for 20 min and isocratic $60 \% \mathrm{~A}, 8 \% \mathrm{~B}, 32 \% \mathrm{C}$ for $5 \mathrm{~min}$.

Method 2 (for the ellagic acid analysis): In order to achieve a better separation of ellagic acid from other phenolic compounds present in fraction $b$ a different HPLC method was developed. The mobile phase was composed of solvent $A$ ( $2.5 \%$ acetic acid in water) and solvent $B(100 \%$ HPLC-grade methanol). The following gradient was used at a flow rate of $0.5 \mathrm{~mL} \mathrm{~min}^{-1}$ : from $100 \%$ A to $50 \%$ A for 50 min. Fraction $c$ was also analysed using this method in order to check the presence of ellagic acid.

In both chromatographic methods detection was carried out at $250 \mathrm{~nm}$ as the preferred wavelength. Spectra were recorded from 220 to $600 \mathrm{~nm}$.

For all fractions, the mass analyses were performed using a Finnigan $^{\mathrm{TM}}$ LCQ ion trap instrument (Thermoquest, San Jose, CA, USA) equipped with an electrospray ionisation (ESI) interface. The LC system was connected to the probe of the mass spectrometer via the UV cell outlet. The optimisation of the conditions was done with a castalagin solution. Both the sheath gas and the auxiliary gas were nitrogen. The sheath gas flow was $1.2 \mathrm{~L} \mathrm{~min}^{-1}$ and the auxiliary gas flow, $6 \mathrm{~L} \mathrm{~min}^{-1}$. The source voltage was $4.5 \mathrm{kV}$. The capillary voltage was $-27 \mathrm{~V}$. Spectra were recorded in negative ion mode between $\mathrm{m} / \mathrm{z} 100$ and $\mathrm{m} / \mathrm{z} 2000$. The mass spectrometer was programmed to do a series of four consecutive scans: a full mass, a zoom mass and an $\mathrm{MS}^{2}$ scan of the most abundant ion in the full mass and an $\mathrm{MS}^{3}$ of the most abundant ion in the MS ${ }^{2}$. The normalised energy of collision was $30 \%$.

\subsection{Analysis of red wine samples}

In order to verify the usefulness of the isolation method in real red wine samples three different wines (A, B and C) were fractionated and their fractions analysed with the same methods as the fractions of the spiked wine. Wine A was made from $V$. vinifera $\mathrm{L}$. cv. "Graciano" grapes and was analysed after 2 months' malolactic fermentation in oak barrels. Wine B was made from $V$. vinifera $\mathrm{L}$. cV. "Tempranillo" grapes and was analysed after ageing 4 months in oak barrels and eight in the bottle. Wine $C$ was made from $V$. vinifera L. cv. "Graciano" grapes and was analysed after ageing 14 months in oak barrels and twelve in the bottle. The wines (de-alcoholised in a rotary evaporator and diluted $3 / 5$ in $2.5 \%$ acetic acid) were also analysed before the fractionation by means of HPLC-DAD-MS (Method 1). 


\section{Results and discussion}

\subsection{Fractionation method}

The HPLC-DAD-MS analysis of the spiked wine before its fractionation revealed the complexity of the sample. Fig. 3 shows its chromatogram (dilution $3 / 5$ in $2.5 \%$ acetic acid) recorded at $250 \mathrm{~nm}$. The elevation of the baseline in the earlier part of the chromatogram and the presence of a "hump" from 20 to 55 min were indicative of the co-elution of a large number of compounds making the detection and identification of the ellagitannins difficult. Since the concentration of the ellagitannins in the spiked wine (see Section 2.2) was twice or more times higher than that reported in wines $[2,22]$, it was possible to localise them in the chromatogram by the mass analysis. Two peaks ( 2 and 4 ) appeared in the extracted ion current chromatogram (EIC) of the signal at $\mathrm{m} / z 933$ and another two (1 and 3 ) in that of the signal at $m / z$ 1065. Taking into account the chromatographic features of the ellagitannins used to spike the wine peak 2 was assigned to vescalagin and peak 4 to castalagin. Similarly, peak 1 corresponded to grandinin and peak 3 to roburin E. However, the co-elution of these compounds with others hindered the obtaining of their spectral features. Furthermore, as the ellagitannin levels in real oak-matured red wines are much lower than in the spiked one it can be easily deduced that the fractionation of the sample is a required step in their determination.

In the present study the procedure proposed by Lee and coworkers [25] for the identification of ellagic acid conjugates in grapes was modified and employed for the identification of oak ellagitannins in oak-matured red wines. The modifications carried out in the method allowed the obtaining of all the oak ellagitannins in the same fraction, isolated from other phenolic compounds.

As shown in Fig. 2 the spiked wine was initially fractionated on a C-18 Sep-Pak ${ }^{\circledR}$ cartridge and three fractions were obtained, one eluting with $2.5 \%$ acetic acid (fraction $a$ ), a second one eluting with ethyl acetate (fraction $b$ ) and a third (fraction $c$ ) eluting with methanol. The HPLC-DAD-MS analysis of the fractions revealed that fraction $a$ contained all the oak ellagitannins and other polar substances, such as gallic acid.

None of the oak ellagitannins was detected in fractions $b$ and $c$ which were initially analysed with method 1 and then with method 2 in order to achieve a better separation of the peak corresponding to ellagic acid, since with method 1 it eluted in the "hump". Ellagic acid was only detected in fraction $b$. In this fraction it was also possible to detect other phenolic compounds, such as the glucosylated derivative of ellagic acid, flavanols (monomeric and oligomeric forms, some of them galloylated) and flavonols (aglycones, glu-

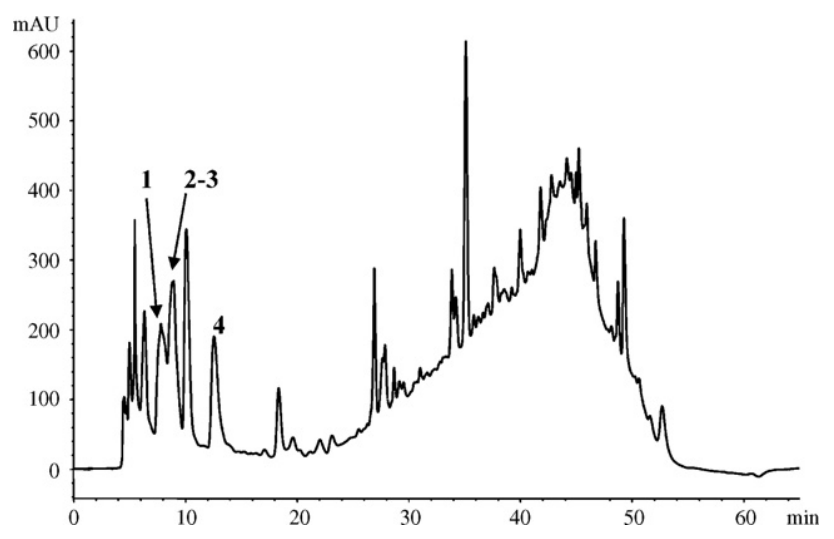

Fig. 3. Chromatogram of the spiked wine before its fractionation recorded at $250 \mathrm{~nm}$. (1) Grandinin, (2) vescalagin, (3) roburin E and (4) castalagin.

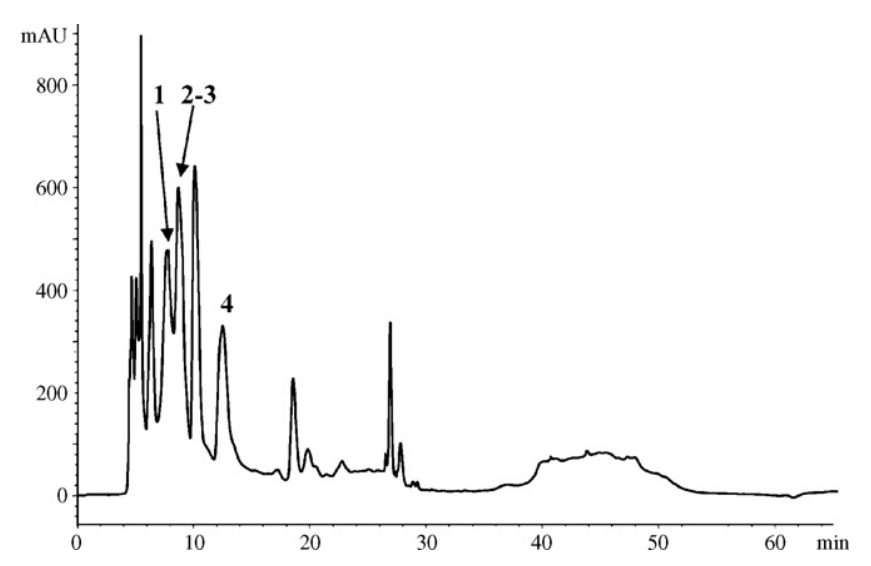

Fig. 4. Chromatogram corresponding to fraction $a$ of the spiked wine recorded at $250 \mathrm{~nm}$. (1) Grandinin, (2) vescalagin, (3) roburin E and (4) castalagin.

cosides and glucuronides of quercetin and myricetin, above all) as well as compounds structurally related to ellagitannins (HHDPglucose).

Fraction $c$ was mostly constituted by anthocyanins (monoglucosides, acetylated and coumaroylated derivatives).

Although all the oak ellagitannins added to the wine sample eluted in fraction $a$, many other compounds still co-eluted with them (Fig. 4). As in the non-fractionated wine the identification of the peaks corresponding to the oak ellagitannins was only achieved from the results of the mass analyses. Thus, another fractionation step was required to improve the separation.

As in the procedure proposed by Lee et al. [23], fraction $a$ was loaded on a hand-packed Sephadex LH-20 minicolumn (Fig. 2). However, in our case, the elution steps were modified in order to obtain a better separation of the targeted compounds. Four fractions were obtained with three different eluents (ultrapure water, ethanol $96 \%$ vol. and methanol) and were analysed by HPLCDAD-MS. The fractions obtained in the elution of fraction $a$ with ultrapure water (fraction 1) and with ethanol (fraction 2) (Fig. 5) contained most of the compounds which interfered with the determination of oak ellagitannins in fraction $a$, since they have similar chromatographic features. The removal of these compounds is an important advantage of the fractionation method developed in this work.

The third fraction (fraction 3) contained, above all, gallic acid (Fig. 6). The HPLC-DAD-MS analysis of these three fractions showed that none of them contained ellagitannins, as in the EIC of the signals at $m / z 933$ and at $m / z 1065$ only noise was detected. The fourth fraction (fraction 4, Fig. 7) contained all the oak ellagitannins, isolated from all the compounds that co-eluted with them. Thus, in this fraction it was possible to detect and identify the four ellagitannins, not only from the data provided by the HPLC-MS analysis (Fig. 7c), which showed two peaks in the EIC at $m / z 933$ (peaks 2 and 4) and another two in that at $m / z 1065$ (peaks 1 and 3), but also from the UV spectra (Fig. 7b) of these peaks, which were typical of this kind of ellagitannins [24].

Initially, fractions 3 and 4 were collected together. However, the presence of gallic acid, that possesses a retention time similar to those of the main oak ellagitannins, interfered in their detection and, above all, in their quantification. In order to avoid this, fraction 3 was eluted with $1 \mathrm{~mL}$ of methanol and fraction 4 was then eluted with five more millilitres of methanol.

Even after this separation and due to the similar chromatographic properties of the analysed ellagitannins, related to the fact of being isomers (vescalagin (2) and castalagin (4); grandinin (1) and roburin E (3), Fig. 1), the chromatogram obtained for fraction 

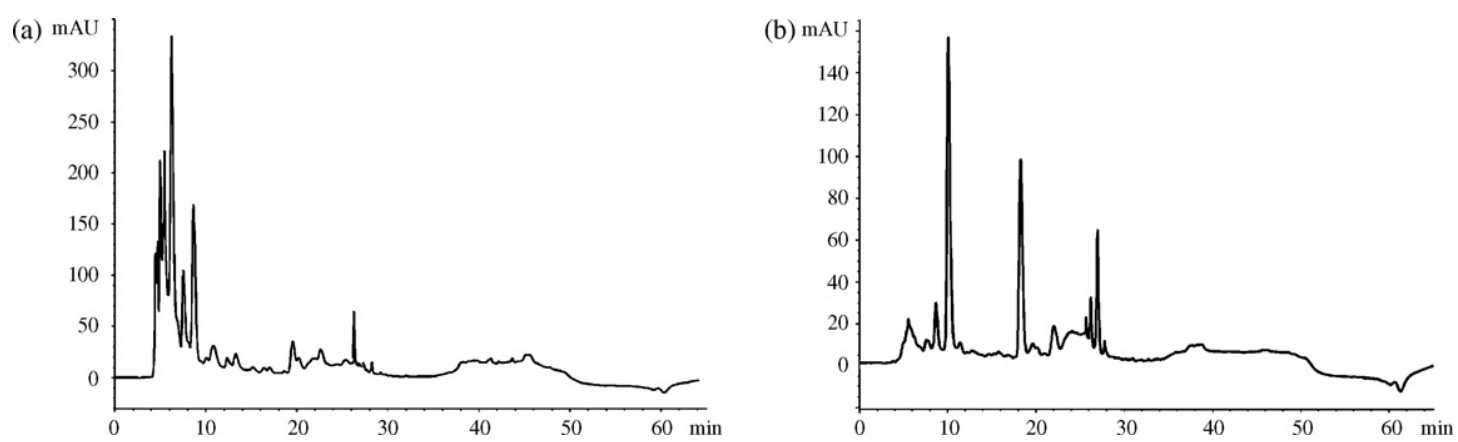

Fig. 5. Chromatograms corresponding to (a) fraction 1 and (b) fraction 2 of the spiked wine recorded at $250 \mathrm{~nm}$.

4 (Fig. 7a) showed a slight overlapping of the peaks of the three first ellagitannins. For this reason accurate quantification of the individual ellagitannins from the UV chromatogram would not be possible. The mass analysis would solve this problem derived from co-elution, since it allows the obtaining of the area of any individual compound. Nevertheless, quantification using mass detection would only be possible if there were an internal standard to correct the high variability of the mass signal. Some authors [22] have proposed chlorogenic acid as an internal standard. However, in our laboratory, and using different kinds of mass spectrometers (ion trap and single quadrupole mass analysers), no stable relationship has been found between the signal of the chlorogenic acid and those of the four ellagitannins studied in this work. This is
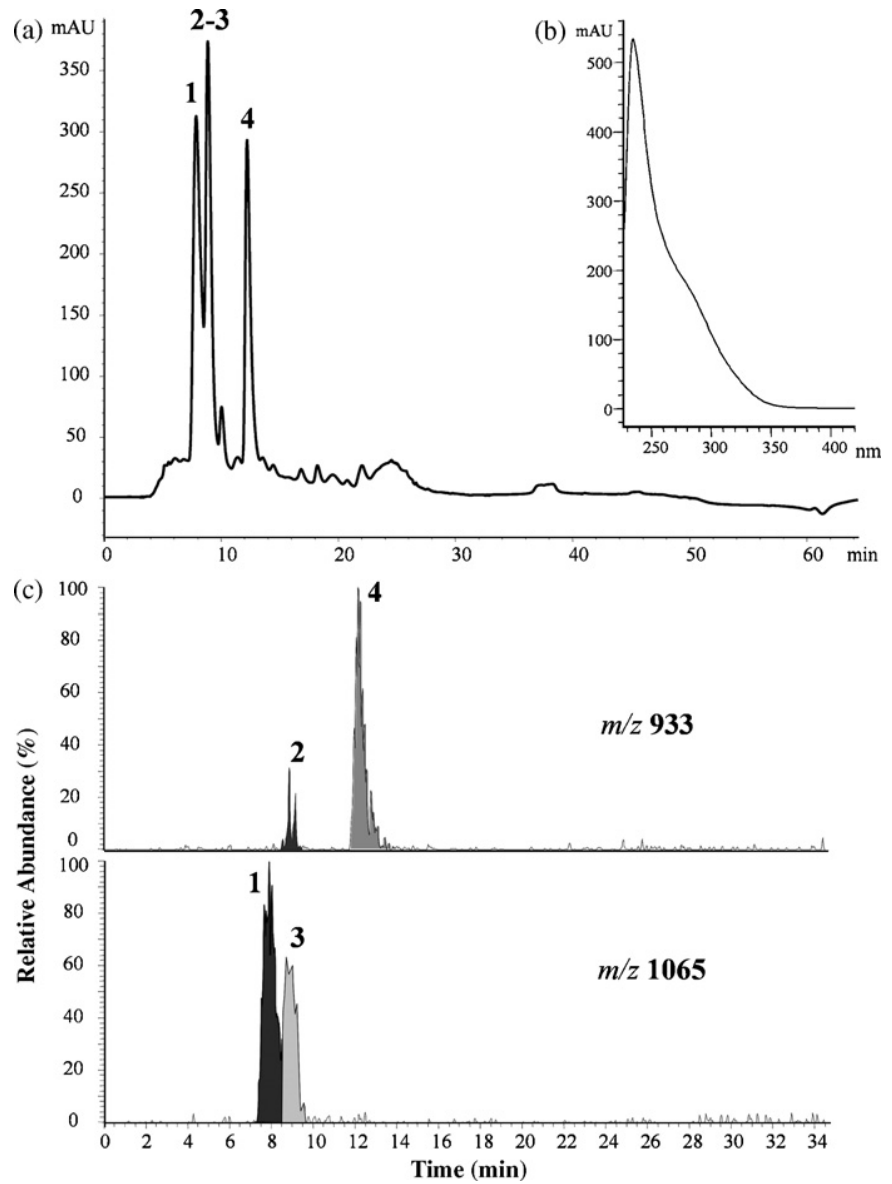

Fig. 7. (a) Chromatogram of fraction 4 of the spiked wine recorded at $250 \mathrm{~nm}$. (b) UV-vis spectra of the oak ellagitannins detected in this fraction. (c) EIC (extracted ion current chromatogram) of the signals at $\mathrm{m} / z 933$ and $\mathrm{m} / z 1065$ in fraction 4 . (1) Grandinin, (2) vescalagin, (3) roburin E and (4) castalagin. probably due to the differences in the molecular structure and properties between ellagitannins and chlorogenic acid. It would be expected that a compound with a molecular structure more similar to those of these ellagitannins would be a better internal standard. Nevertheless, the candidates to be used as internal standards are mostly found in natural sources whose obtaining either by extraction-purification or by hemi-synthetic method is complicated and time-consuming.

\subsection{Recovery of the fractionation method}

The recovery was calculated from the application of this separation method in triplicate to a wine sample before and after the addition of known amounts of pure castalagin (50\%, 100\% and $150 \%$ of the content reported for this ellagitannin in wine [22]) and from the comparison between the amount determined in the ellagitannin-rich fraction (fraction 4, see Fig. 2) and the added one. As the peak of the castalagin was clearly separated from others in the chromatogram of this fraction recorded at $250 \mathrm{~nm}$ it was possible to quantify it from the area of the peak using an external calibration curve of castalagin. The mean recovery, which takes into account the mean recovery at each concentration and the mean of these recoveries, was $91.5 \%$.

\subsection{Application of the fractionation method to oak-matured red wines}

Once tested with the spiked wine the developed method was used to analyse the oak ellagitannins in three real wines in distinct stages of the winemaking and ageing. Differences in the

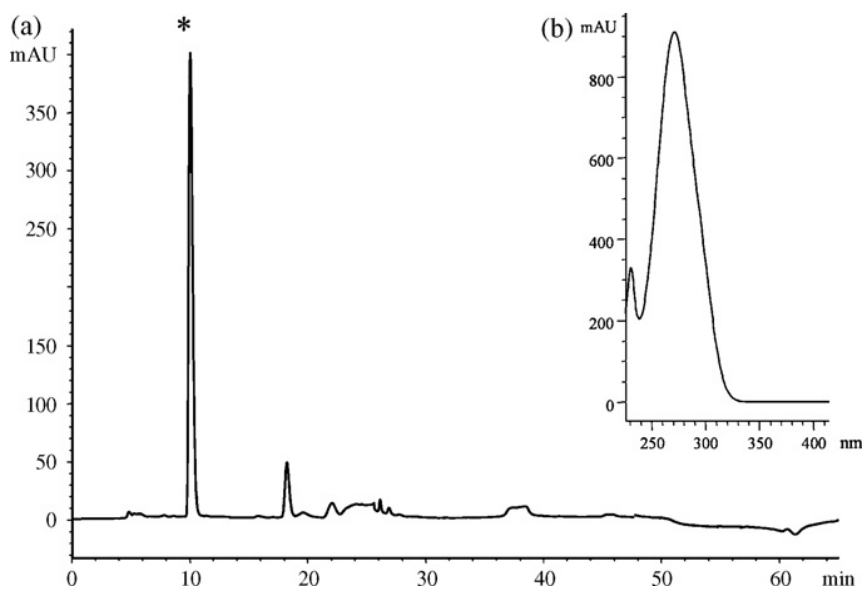

Fig. 6. (a) Chromatogram of fraction 3 of the spiked wine recorded at $250 \mathrm{~nm}$. The peak with the asterisk $\left({ }^{*}\right)$ corresponds to gallic acid. (b) UV-vis spectrum of gallic acid. 

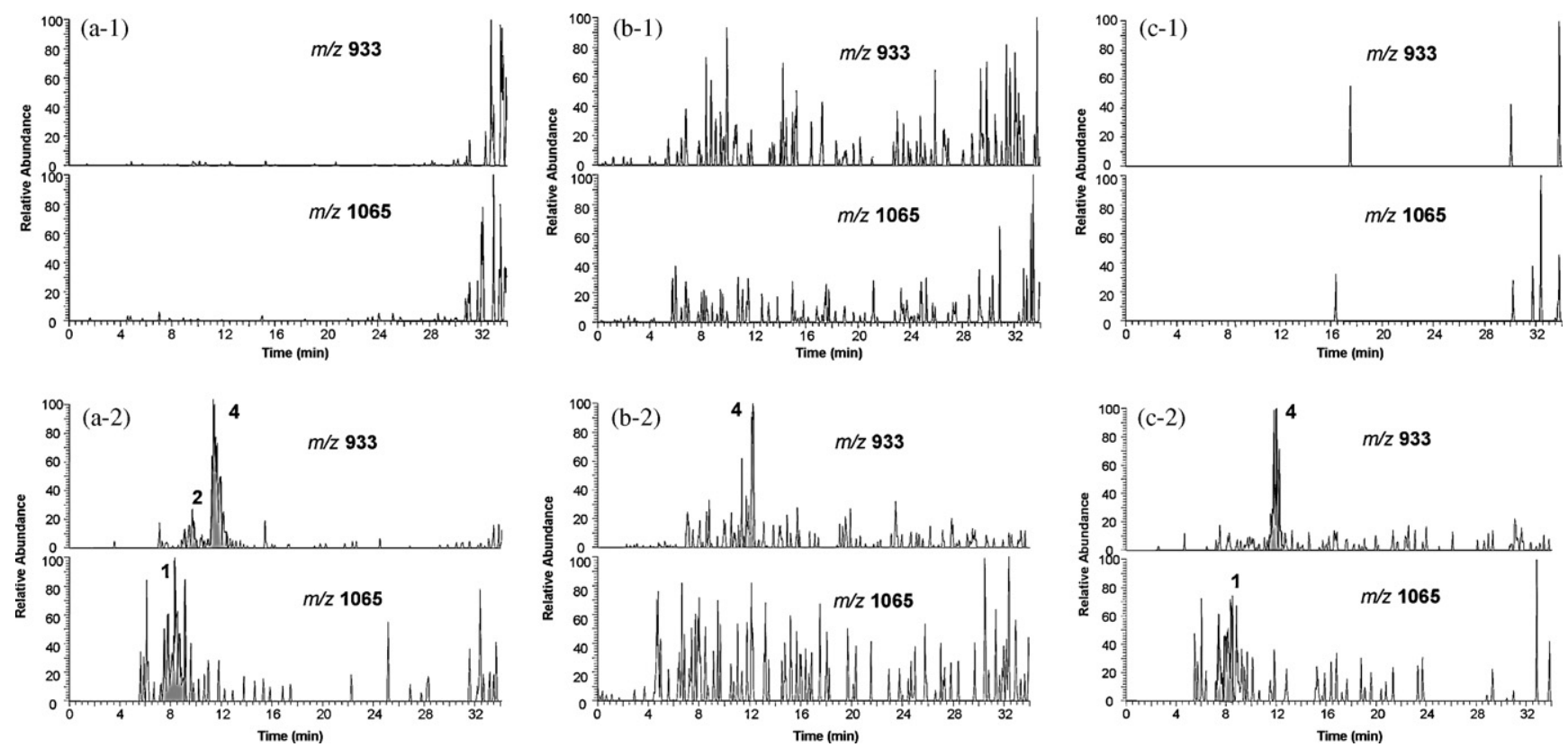

Fig. 8. EIC of the signals at $m / z 933$ and $m / z 1065$ in wines A (a), B (b) and C (c), before fractionation (a-1, b-1, c-1) and in the corresponding fractions 4 (a-2, b-2, c-2) obtained after fractionation of the samples. (1) Grandinin, (2) vescalagin and (4) castalagin.

ellagitannin levels of these three wines were expected due to the differences in their ages and in the time spent in oak barrels. None of the targeted ellagitannins was detected in the wines when analysed directly after de-alcoholisation and dilution of the sample. However, when these wines were fractionated, it was possible to detect some ellagitannins in fraction 4 (Fig. 8).

In the case of wine A (wine after 2 months of malolactic fermentation in oak barrels), grandinin, vescalagin and castalagin could be detected. For wine $\mathrm{B}$ (wine after ageing 4 months in oak barrels and 8 in the bottle), only castalagin could be detected. In the third case, wine $C$ (wine after ageing 14 months in oak barrels and 12 in the bottle), the developed method allowed the detection of grandinin and castalagin.

\section{Conclusions}

A two-step fractionation method consisting of a solid phase extraction in C-18 Sep-Pak ${ }^{\circledR}$ cartridges followed by size exclusion chromatography in a hand-packed Sephadex LH-20 minicolumn has been optimised for the detection and identification of the main oak ellagitannins in red wine samples. Oak ellagitannins were only present in one fraction and separated from other phenolic compounds that might interfere in the HPLC-DAD-MS analyses of the fractions, such as, for example, gallic acid which behaves similarly to ellagitannins in reversed-phase. The use of this method in wine samples corresponding to different stages of the winemaking and ageing and produced from grapes belonging to different cultivars has allowed the detection of grandinin, vescalagin and castalagin in them. Furthermore, this isolation method has additional advantages: the low amount of wine sample required for the analysis $(3 \mathrm{~mL})$ and the short time required. The chromatographic method developed for the analyses of the fractions has allowed the separation of compounds with related structures and pseudo-molecular ions with identical $\mathrm{m} / \mathrm{z}$ ratios which makes their individual detection, identification and, in the future, quantification possible provided a suitable internal standard is found.

\section{Acknowledgements}

Thanks are due to the Spanish MICINN and FEDER (Grant Ref. AGL2008-05569-C02-01) for financial support. The authors also thank the Spanish MICINN for an F.P.U. predoctoral scholarship to I. García-Estévez and Mr. G.H. Jenkins for his help with the English version of the ms.

\section{References}

[1] N. Vivas, Y. Glories, Am. J. Enol. Vitic. 47 (1996) 103.

[2] A. Glabasnia, T. Hofmann, J. Agric. Food Chem. 54 (2006) 3380.

[3] J.L. Puech, F. Feuillat, J.R. Mosedale, Am. J. Enol. Vitic. 50 (1999) 469.

[4] A. Scalbert, B. Monties, J.M. Favre, Phytochemistry 27 (1988) 3483.

[5] W. Mayer, W. Gabler, A. Riester, H. Korger, Liebigs Ann. Chem. 707 (1967) 177.

[6] C.L.M. Hervé du Penhoat, V.M.F. Michon, A. Ohassan, S. Peng, A. Scalbert, D. Gage, Phytochemistry 30 (1991) 329.

[7] C.L.M. Hervé du Penhoat, V.M.F. Michon, S. Peng, C. Viriot, A. Scalbert, D. Gage, J. Chem. Soc., Perkin Trans. 1 (1991) 1653.

[8] B. Fernández de Simón, T. Hernández, E. Cadahía, M. Dueñas, I. Estrella, Eur. Food Res. Technol. 216 (2003) 150.

[9] J.R. Mosedale, J.L. Puech, F. Feuillat, Am. J. Enol. Vitic. 50 (1999) 503.

[10] J. Marco, J. Artajona, M.S. Larrechi, F.X. Rius, Am. J. Enol. Vitic. 45 (1994) 192.

[11] G. Masson, M. Moutounet, J.L. Puech, Am. J. Enol. Vitic. 46 (1995) 262.

[12] M.D. Hale, K. McCafferty, E. Larmie, J. Newton, J.S. Swan, Am. J. Enol. Vitic. 50 (1999) 495.

[13] L. Matricardi, A.L. Waterhouse, Am. J. Enol. Vitic. 50 (1999) 519.

[14] S. Peng, A. Scalbert, B. Monties, Phytochemistry 30 (1991) 775.

[15] C. Viriot, A. Scalbert, C. Lapierre, M. Moutounet, J. Agric. Food Chem. 41 (1993) 1872.

[16] M. Del Alamo-Sanza, I. Nevares-Domínguez, Anal. Chim. Acta 563 (2006) 255.

[17] M. Del Alamo-Sanza, J.A. Fernández-Escudero, R. De Castro-Torío, Food Sci. Technol. Int. 10 (2004) 233.

[18] C. Santos-Buelga, A. Scalbert, J. Sci. Food Agric. 80 (2000) 1094

[19] S. Quideau, K.S. Feldman, Chem. Rev. 96 (1996) 475.

[20] J.H. Lee, S. Talcott, J. Agric. Food Chem. 50 (2002) 3971.

21] M.F. Nonier, N. Vivas, N. Vivas de Gaulejac, C. Absalon, C. Vitry, E. Fouquet, J. Sci. Food Agric. 85 (2005) 343.

[22] C. Saucier, M. Jourdes, Y. Glories, S. Quideau, J. Agric. Food Chem. 54 (2006) 7349.

[23] J.H. Lee, J.V. Johnson, S.T. Talcott, J. Agric. Food Chem. 53 (2005) 6003.

[24] N. Vivas, M.F. Nonier, N. Vivas de Gaulejac, I. Pianet de Boissel, C. R. Chim. 7 (2004) 945.

[25] D. Fridrich, A. Glabasnia, J. Fritz, M. Esselen, G. Pahlke, T. Hofmann, D. Marko, J. Agric. Food Chem. 56 (2008) 3010. 